\title{
Salvage endoscopic submucosal dissection for incompletely resected esophageal or gastric neoplasms (case series)
}

\section{다)(1) $\odot$}

Authors

Satoshi Kinoshita ${ }^{1,2}$, Toshihiro Nishizawa ${ }^{1,2,3}$, Yasutoshi Ochiai ${ }^{2}$, Ai Fujimoto ${ }^{1,2}$, Kaoru Takabayashi ${ }^{4}$, Motohiko Kato², Osamu Goto ${ }^{1,5}$, Tadateru Maehata ${ }^{2}$, Naohisa Yahagi², Toshio Uraoka',6

Institutions

1 Department of Gastroenterology, National Hospital Organization Tokyo Medical Center

2 Division of Research and Development for Minimally Invasive Treatment, Cancer Center, Keio University School of Medicine

3 Department of Gastroenterology and Hepatology, International University of Health and Welfare Mita Hospital

4 Center for Diagnostic and Therapeutic Endoscopy, School of Medicine, Keio University, Tokyo, Japan

5 Department of Gastroenterology, Nippon Medical School

6 Department of Internal Medicine, Division of Gastroenterology and Hepatology, Gunma University, Gunma, Japan

submitted 19.12.2018

accepted after revision 11.3.2019
Bibliography

DOI https://doi.org/10.1055/a-0998-3917 |

Endoscopy International Open 2020; 08: E20-E24

(c) Georg Thieme Verlag KG Stuttgart · New York elSSN 2196-9736

Corresponding author Toshio Uraoka, MD, PhD, Department of Gastroenterology and Hepatology, Gunma University Graduate School of Medicine, 3-39-15 Showamachi, Maebashi-shi, Gunma 371 8511 , Japan

Fax: +81-3-3412-9811

toshi_urao@yahoo.co.jp

\section{ABSTRACT}

Background and study aims Given that positive lateral margins indicate possible residual carcinoma, salvage endoscopic submucosal dissection (ESD) should be considered for curative therapy. Presence of submucosal fibrosis, however, makes the procedure difficult to perform. We present our case series to discuss the feasibility of salvage ESD and the timing of the procedure.

\section{Introduction}

Endoscopic submucosal dissection (ESD) is now performed all around the world. Advances in the procedure have enabled endoscopic en bloc resection of lesions regardless of their or site or presence of ulcer scars [1]. To achieve curative resection, it is essential to accurately determine the margin of the neoplasm before performing the ESD. Magnifying image-enhanced endoscopy and chromoendoscopy are widely used to determine the appropriate tumor margin in advance of the procedure [2], but precise results can be difficult to obtain. Hence, positive lateral margins of a neoplasm can still occur after ESD. Salvage ESD should be considered in such cases, as positive lateral margins indicate possible residual carcinoma. The chief challenge in performing a salvage ESD is presence of fibrosis at the site where the submucosal layer has been eliminated by the previous ESD. No consensus has been reached on timing of sal- vage ESD. We present our case series to discuss the feasibility of salvage ESD and the timing of the procedure.

\section{Case reports}

\section{Patients}

Six patients who underwent salvage ESD for residual tumors between January 2015 and October 2018 were enrolled in this study. Consecutive patients were identified from a computerized database listing all patients who underwent endoscopic procedures at Keio University Hospital and the National Hospital Organization Tokyo Medical Center. Four cases had early gastric cancer and two cases had superficial esophageal cancer. The salvage ESDs were performed after histological confirmation of positive lateral margins in the initial ESD specimens. 
- Table 1 Clinical characteristics of patients.

\begin{tabular}{|c|c|c|c|c|c|c|}
\hline & Case 1 & Case 2 & Case 3 & Case 4 & Case 5 & Case 6 \\
\hline Gender & Male & Female & Male & Male & Male & Male \\
\hline Age & 77 & 82 & 65 & 67 & 78 & 68 \\
\hline Location & Esophagus & Stomach & Stomach & Esophagus & Stomach & Stomach \\
\hline Days after first ESD & 15 & 21 & 35 & 104 & 210 & 247 \\
\hline Histological type & $\begin{array}{l}\text { Squamous cell } \\
\text { carcinoma }\end{array}$ & $\begin{array}{l}\text { Tubular adeno- } \\
\text { carcinoma }\end{array}$ & $\begin{array}{l}\text { Tubular adeno- } \\
\text { carcinoma }\end{array}$ & $\begin{array}{l}\text { Squamous cell } \\
\text { carcinoma }\end{array}$ & $\begin{array}{l}\text { Tubular adeno- } \\
\text { carcinoma }\end{array}$ & $\begin{array}{l}\text { Tubular adeno- } \\
\text { carcinoma }\end{array}$ \\
\hline Residual lesion size (mm) & $5 \times 3$ & $1.5 \times 1.5$ & $30 \times 23$ & 0 & $15 \times 12$ & $19 \times 5$ \\
\hline Resection size (mm) & $15 \times 12$ & $35 \times 35$ & $50 \times 35$ & $90 \times 30$ & $25 \times 20$ & $40 \times 17$ \\
\hline Procedure time (min) & 45 & 52 & 136 & 70 & 65 & 65 \\
\hline En bloc resection & + & + & + & + & + & +- \\
\hline Fibrosis & F0 & F1 & $\mathrm{F} 2$ & $\mathrm{~F} 2$ & $\mathrm{~F} 2$ & $\mathrm{~F} 2$ \\
\hline Complication & - & - & - & - & - & - \\
\hline
\end{tabular}

\section{ESD procedures}

ESD procedures were performed using video endoscopy (GIFQ260 J or GIF-H260Z; Olympus Co, Tokyo, Japan). The border of a residual lesion was determined by magnifying image-enhanced endoscopy and chromoendoscopy. The safety margin was set as $3 \mathrm{~mm}$ or more. For ESD-induced ulcers in the scarring stage, the residual lesion was resected together with the scar. Open ESD-induced ulcers were not included in the resected area. The ESD was performed using a DualKnife (Olympus) and a transparent hood (Olympus) attached to the tip of the endoscope to obtain a good endoscopic view of the submucosal layer and facilitate dissection [3]. A mucosal incision was made after administering a submucosal injection of Glycerol (Chugai Pharmaceutical, Tokyo, Japan) with small amounts of indigo carmine and epinephrine added. More Glycerol was then injected into the submucosal layer to lift the lesion, and the thickened submucosal layer was dissected. A HookKnife (Olympus) was also used when fibrosis in the submucosal layer was severe. Hyaluronic acid solution also was injected into the submucosal layer when submucosal fibrosis impeded sufficient lifting. $\mathrm{CO}_{2}$ insufflation was performed during all procedures. Patients undergoing gastric ESD were sedated with midazolam and pethidine; patients undergoing esophageal ESD were sedated with midazolam, pethidine, and dexmedetomidine. Salvage ESDs were performed by four experienced endoscopists, each of whom had performed more than 200 ESDs. The resected specimens were pathologically assessed by experienced pathologists. An R0 resection was defined as en bloc resection with no remaining lateral or vertical margins. Degree of submucosal fibrosis was classified into three groups based on findings observed at the time of submucosal local injection: $F 0$, no fibrosis, which manifested as a transparent layer; F1, mild fibrosis, which appeared as a white, web-like structure in the submucosal layer; and F2, severe fibrosis, which appeared as a white muscular structure without a transparent layer in the submucosal layer
[4]. Procedure time was defined as time from incision until complete removal of the lesion.

All specimens were examined to determine tumor size, macroscopic type, histological type, depth of invasion, lateral resection margin, and vertical resection margin.

All six patients have undergone follow-up endoscopies once a year since the salvage ESDs.

\section{Patient characteristics}

Characteristics of the six patients are shown in $>$ Table 1.

Days elapsed after the first ESD were 15, 21, 35, 104, 210 and 247, respectively. Degree of fibrosis during ESD was F0 (none) in Case 1, F1 (mild) in Case 2 and F2 (severe) in Cases 3 to 6 ( $\mathbf{F i g . 1}$ ). En bloc resection was achieved in all cases, and no instances of postoperative bleeding or intraoperative perforation have occurred in any case. No local recurrence was observed after salvage ESD. Median follow-up time was 301 days (range $14-570$ days).

\section{Superficial esophageal cancer case}

Case 1 was a 77 -year-old male patient ( $\bullet$ Fig. 2 ) in whom a superficial esophageal cancer, Type $0-I I$, measuring $12 \mathrm{~mm}$ in diameter, was found in the middle of the thoracic esophagus. ESD was performed after Lugol staining. Histological examination of the resected specimen revealed intramucosal squamous cell carcinoma without lymphatic or vascular involvement. The vertical margin was negative, but the horizontal margin was positive. Salvage ESD was performed 15 days later to remove any possible residual carcinoma. No fibrosis was observed in the submucosal layer during the 45 -minute procedure (F0). Histological examination of the resected specimen revealed highgrade intraepithelial neoplasia without lymphatic or vascular involvement. The vertical and horizontal margins were both pathologically negative. 

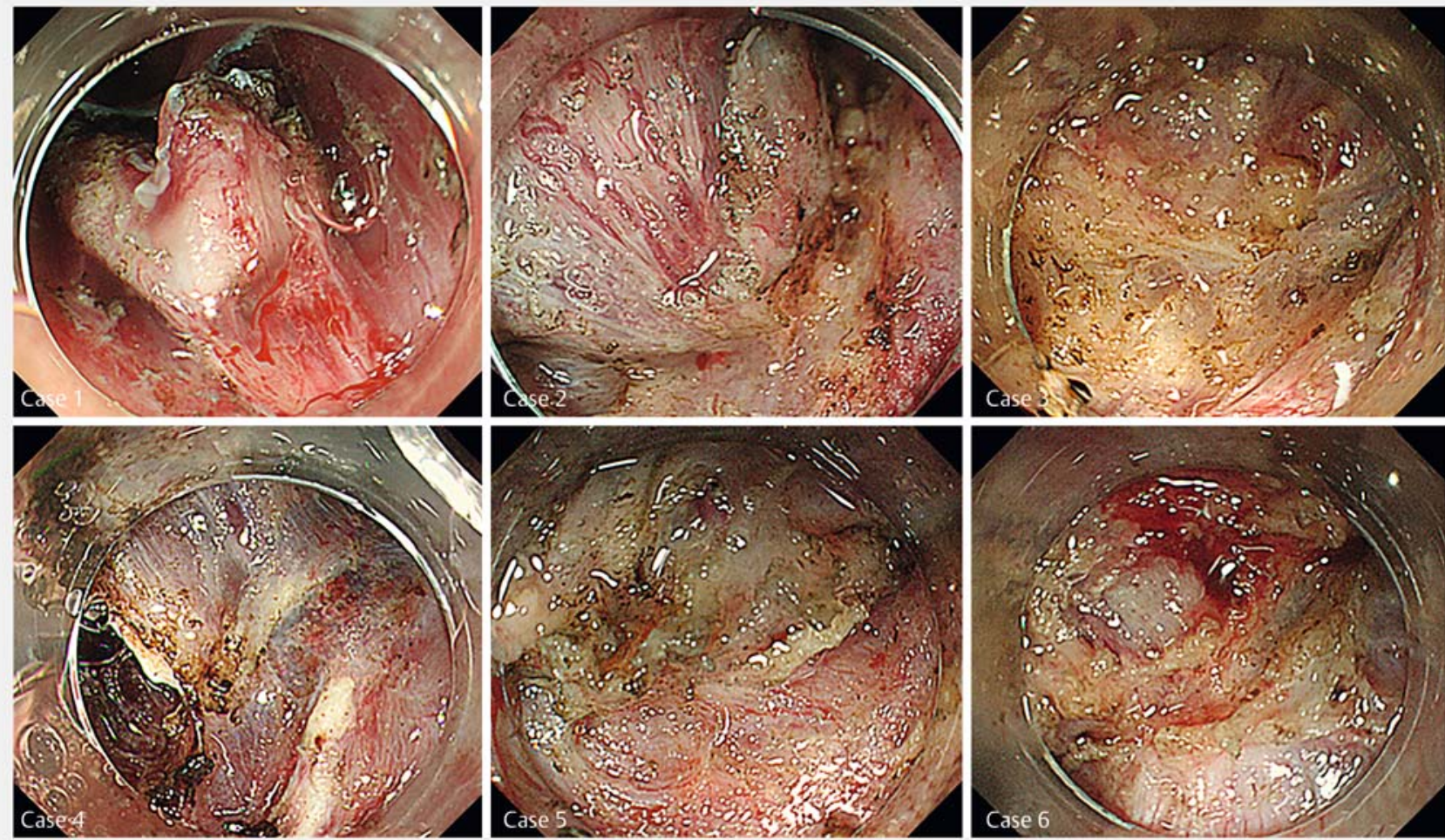

Fig. 1 Degree of fibrosis during ESD. Case 1, F0 (none); Case 2, F1 (mild); Cases 3 -6, F2 (severe).
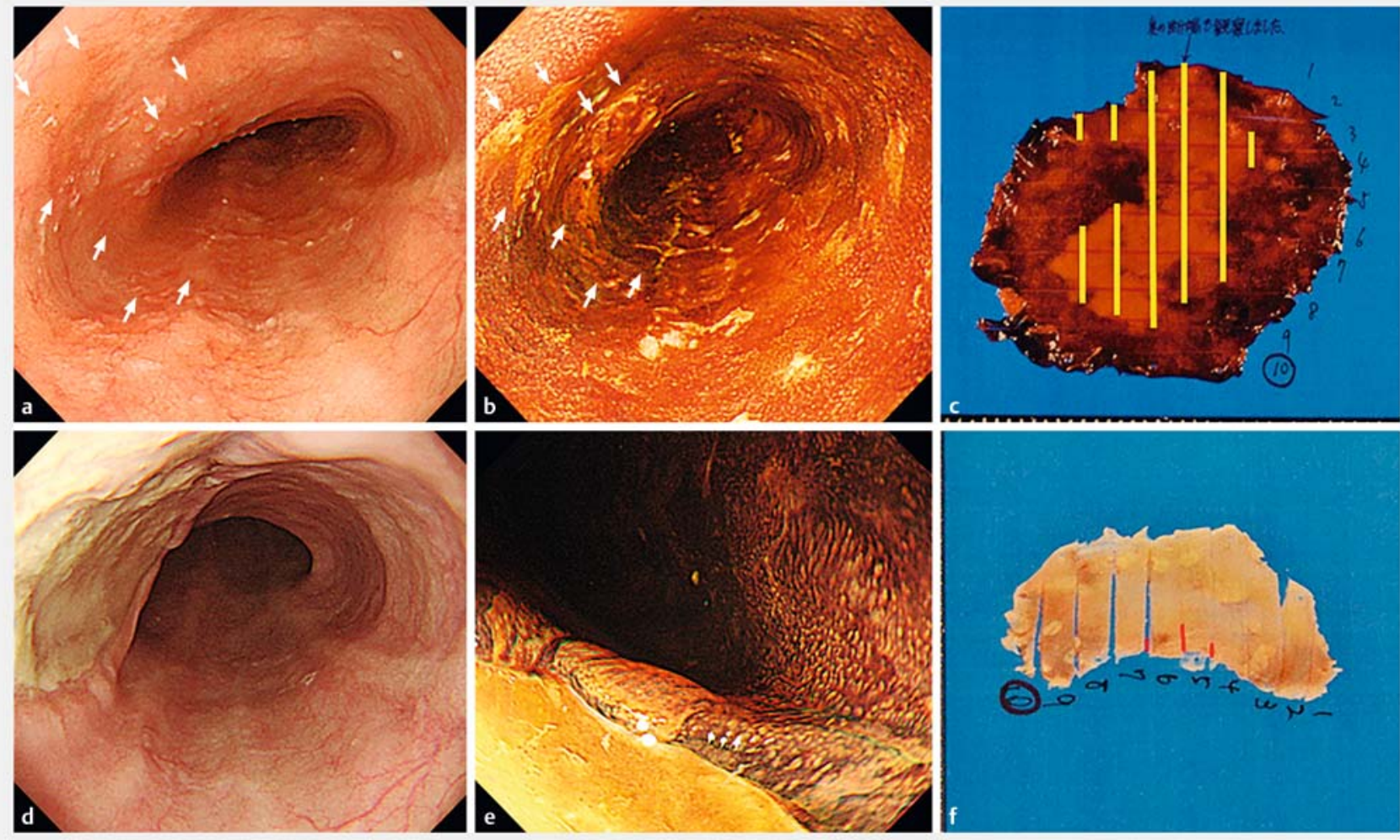

- Fig. 2 Case 1: superficial esophageal cancer. a A type 0-IIc superficial esophageal cancer was observed in the middle of the thoracic esophagus. Arrows indicate the lesion. $\mathbf{b}$ Lugol staining. $\mathbf{c}$ Histological examination of the resected specimen revealed intraepithelial squamous cell carcinoma with a positive horizontal margin. $\mathbf{d}$ ESD-induced ulcer. e Lugol staining. $\mathbf{f}$ We performed salvage ESD after 15 days. Vertical and horizontal margins were both negative. 

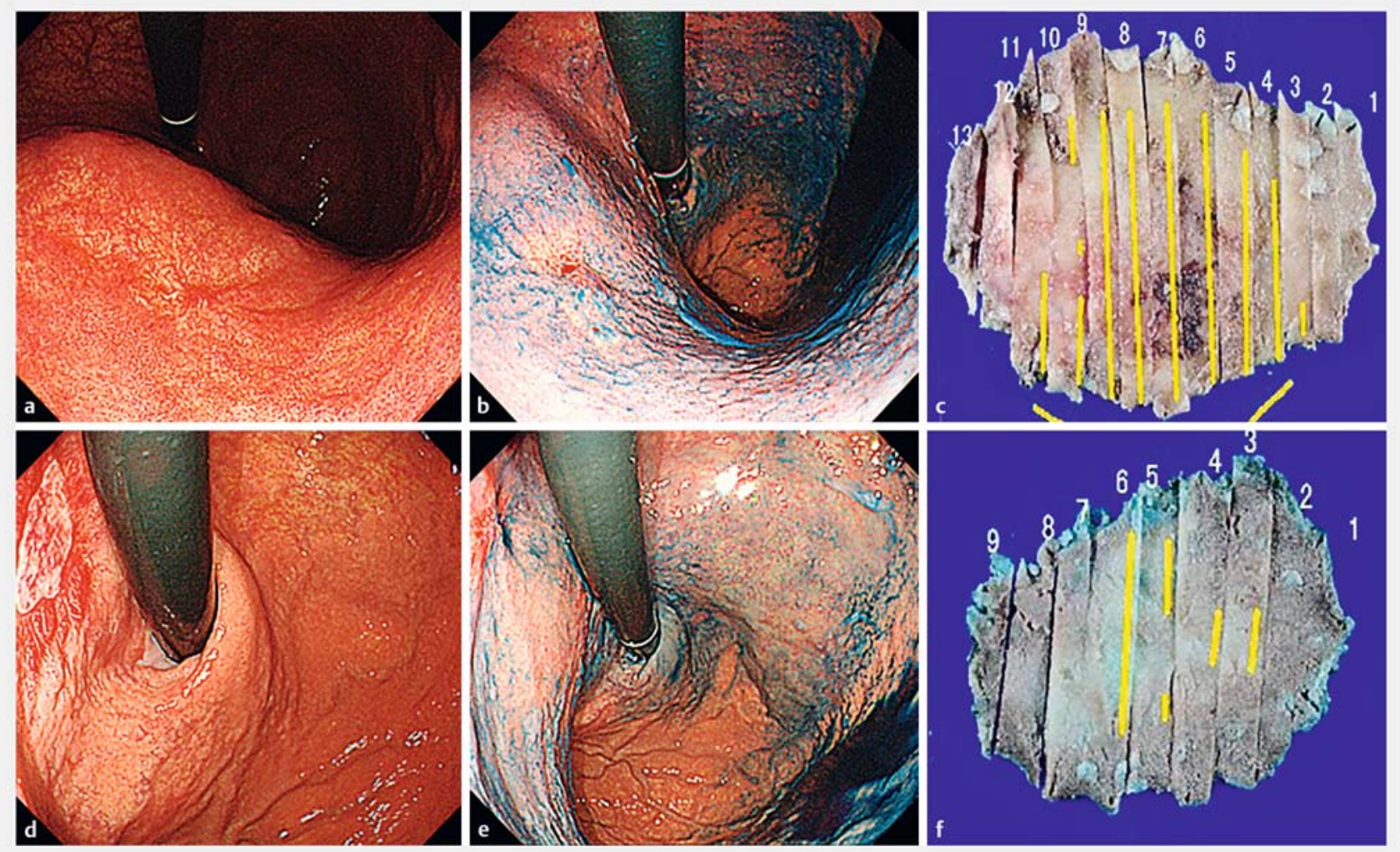

- Fig. 3 Case 3: early gastric cancer. a A superficial elevated 24-mm lesion was observed in the lesser curvature of the upper stomach. b Acetic acid-indigo carmine chromoendoscopy. $\mathbf{c}$ Histological examination of the resected specimen revealed a well-differentiated mucosal adenocarcinoma with a positive horizontal margin. $\mathbf{d}$ ESD-induced ulcer. e Acetic acid-indigo carmine chromoendoscopy. $\mathbf{f}$ We performed salvage ESD after 35 days. Vertical and horizontal margins were both negative.

\section{Early gastric cancer case}

Case 3 was a 65-year-old female patient with early gastric cancer ( $\triangleright$ Fig. 3). A superficial elevated lesion $24 \mathrm{~mm}$ in diameter was observed in the lesser curvature of the upper stomach. Histological examination of the ESD resected specimen revealed a well-differentiated mucosal adenocarcinoma without lymphatic or vascular involvement. The vertical margin was negative but the horizontal margin was positive. A salvage ESD was performed 35 days day later to remove any possible residual carcinoma. Severe fibrosis was observed in the submucosal layer (F2) of the 136-minute procedure. Histological examination of the resected specimen revealed a well-differentiated mucosal adenocarcinoma measuring $30 \mathrm{~mm}$ in diameter, with no lymphatic or vascular involvement. The vertical and horizontal margins were both negative.

\section{Discussion}

Precise determination of tumor margin is sometimes difficult, because the background mucosa can be influenced by inflammation. Lesions with a wide-spreading area have been reported to be a risk factor for residual tumor after ESD for superficial esophageal cancer [5]. Reported risk factors for positive lateral margins in early gastric cancer are lesions with a flat spreading area, lesions with lateral submucosal cancer extension beneath non-cancerous mucosa, and lesions with unexpected nearby lesions [6]. Endoscopists should be wary of unexpected lateral extension and the appearance of multiple lesions at the same time.

Positive lateral margins indicate possible residual carcinoma. Sekiguchi et al. [7] demonstrated that a cancer-positive lateral margin length greater than $6 \mathrm{~mm}$ is a useful predictive factor for local recurrence after noncurative ESD with a positive lateral margin. An early salvage ESD may be technically more acceptable than ESD performed for a local recurrence at a later point of time.

Jeong et al. [8] have shown that submucosal fibrosis may be predictive of prolonged ESD. Sato et al. [9], meanwhile, have reported that presence of fibrosis is associated with difficult ESD procedures and low rates of complete resection. The perforation rate was significantly higher in cases with accompanying fibrosis than in cases without fibrosis [4]. Early salvage ESD before the appearance and spread of submucosal fibrosis therefore merits consideration.

Optimal timing between initial ESD and salvage ESD remains undefined. Han et al. demonstrated that EMR delayed by more than 21 days from the initial biopsy was a significant risk factor of submucosal fibrosis, which suggests that a longer interval 
between diagnostic biopsies and EMR may lead to submucosal fibrosis [10]. Our study was similar to that by Han et al., and the two of our patients who underwent salvage ESD within 21 days were rated as F0 (no fibrosis) and F1 (mild fibrosis).

The retrospective design of this study was a limitation. Moreover, the number of cases was too small to support a recommendation of salvage ESD within 21 days. A further study with a large number of cases should be conducted to learn more about the clinical outcomes and define the golden time for salvage ESD after initial ESD.

\section{Conclusion}

Salvage ESD by experienced endoscopists seems feasible. Early timing might be a promising option when salvage ESD is performed.

\section{Competing interests}

None

\section{References}

[1] Kikuchi D, lizuka T, Hoteya S et al. Safety and efficacy of secondary endoscopic submucosal dissection for residual gastric carcinoma after primary endoscopic submucosal dissection. Digestion 2012; 86: $288-293$

[2] Jung DH, Youn YH, Kim JH et al. Secondary endoscopic submucosal dissection for locally recurrent or incompletely resected gastric neoplasms. World J Gastroenterol 2018; 24: 3776-3785

[3] Nishizawa T, Yahagi N. Endoscopic mucosal resection and endoscopic submucosal dissection: technique and new directions. Curr Opin Gastroenterol 2017; 33: 315-319

[4] Kim EK, Han DS, Ro Y et al. The submucosal fibrosis: what does it mean for colorectal endoscopic submucosal dissection? Intest Res 2016; 14: $358-364$

[5] Wen J, Linghu E, Yang Y et al. Relevant risk factors and prognostic impact of positive resection margins after endoscopic submucosal dissection of superficial esophageal squamous cell neoplasia. Surg Endosc 2014; 28: $1653-1659$

[6] Kakushima N, Ono H, Tanaka M et al. Factors related to lateral margin positivity for cancer in gastric specimens of endoscopic submucosal dissection. Dig Endosc 2011; 23: 227-232

[7] Sekiguchi M, Suzuki H, Oda I et al. Risk of recurrent gastric cancer after endoscopic resection with a positive lateral margin. Endoscopy 2014; 46: $273-278$

[8] Jeong JY, Oh YH, Yu YH et al. Does submucosal fibrosis affect the results of endoscopic submucosal dissection of early gastric tumors? Gastrointest Endosc 2012; 76: 59-66

[9] Sato K, Ito S, Kitagawa T et al. Factors affecting the technical difficulty and clinical outcome of endoscopic submucosal dissection for colorectal tumors. Surg Endosc 2014; 28: 2959-2965

[10] Han KS, Sohn DK, Choi DH et al. Prolongation of the period between biopsy and EMR can influence the nonlifting sign in endoscopically resectable colorectal cancers. Gastrointest Endosc 2008; 67: 97-102 